\title{
Design and Implementation of a General Tracking Controller for Friction Stir Welding Processes
}

\author{
Thomas Oakes and Robert G. Landers \\ Department of Mechanical and Aerospace Engineering \\ Missouri University of Science and Technology \\ 400 West $13^{\text {th }}$ Street, Rolla, Missouri 65409-0050 \\ \{tmo6w3,landersr\}@mst.edu
}

\begin{abstract}
This paper establishes a method for force filtering, develops a dynamic process model, and designs and implements a general tracking controller to regulate the axial force for a variety of reference signals in Friction Stir Welding processes. Steady state and dynamic models are used to relate the input process parameters to the axial force. The general tracking controller is implemented in a Smith Predictor-Corrector Structure to compensate for a pure communication delay. The controller successfully performs bead-on-plate welds using a 6061 aluminum alloy. Both constant and sinusoidal reference forces are tracked.
\end{abstract}

\section{INTRODUCTION}

Friction Stir Welding (FSW) is a solid state joining process that utilizes gross plastic deformation rather than a conventional welding flame to join material. The FSW process is unique in that it can be used to successfully join materials such as aluminum alloys that are difficult to join with other welding processes.

Then FSW joining process requires a nonconsumable tool, containing a shoulder and profiled pin region. The tool is plunged into the part at a specified spindle speed and plunge rate until the shoulder makes contact with the material to be joined. Following a brief dwell period, the rotating tool advances along the weld path at a specified traverse rate and spindle rotation speed. The combination of heat input and tool geometry cause the material along the boundaries of the weld region to deform and mix together to form a solid joint. A process schematic is shown in Figure 1.

Three common process parameters for the FSW process are traverse rate, $v$, spindle speed, $\omega$, and plunge depth, $d$. Constant process parameter runs can result in poor quality welds due to improper fixturing of the work piece, machine geometric errors, and material inconsistencies (i.e., part slope) along the weld path. Cederqvist et al. [2008] fixed values of traverse rate and plunge depth and adjusted the spindle speed online with a Proportional plus Integral plus Derivative controller to regulate the tool pin temperature. The method is effective, but the closed-loop response is sluggish due to the inherent low bandwidth of thermal systems. Zhao et al. [2007] conducted a FSW process with constant traverse rate and spindle speed while varying the plunge depth to control the axial force. A polynomial pole placement technique was used to design a controller based on a desired characteristic equation. The controller was designed specifically to reject constant disturbances and allow tracking of constant axial forces. Kalya [2007] regulated the axial force in a similar manner using an adaptive neural network controller to account for variations in the model dynamics. The axial force is regulated in this paper to ensure the tool maintains proper contact with the part to avoid creating excessive flash and defects such as surface voids and wormholes.

\section{EXPERIMENTAL SYSTEM AND FILTERING}

An ABB IRB 940 Tricept Robot is retrofitted with a FSW spindle head to provide the desired rotational motion. The spindle is driven by a SLM115-368 servo motor and is rated at $10 \mathrm{hp}$ with a range of $\pm 3000 \mathrm{rpm}$. A six axis force/moment sensor (JR3 Inc. model 75E20S$\mathrm{M} 125 \mathrm{~A}-\mathrm{A} 6000 \mathrm{~N} 1150$ ) is used to record the lateral, normal and axial forces, as well as the respective moments. The physical setup is shown in Figure 2. The robot control unit is programmed through RAPID, which allows the operator to pre program the system motion and control algorithms. The code is typed in text format and uploaded to the control unit. Code is typically divided into subsections including: low-level formatting, primary welding loop, interrupt subroutines, and data collection. A teach pendant is used to load the file, select a welding vector and execute the code.

Tool eccentricity and sensor noise contribute to a large variance in the measured force signals; therefore, a first order stochastic process model is used in conjunction with a two-step Kalman filter to effectively reduce the axial force signal variance while preserving the phase and magnitude. The stochastic process model is

$$
f(k)=e^{-\lambda T} f(k-1)+w(k)
$$

where $f$ is the axial force, $k$ is the current iteration, $T$ is the sample period, $w$ is the process noise, and $\lambda$ is a filter tuning parameter. Previous studies have found that $\lambda=0$ provides the best filtering capability in terms of steadystate behavior. The process measurement is

$$
y(k)=F(k)+v(k)
$$


where $F$ is the measured axial force and $v$ is the measurement noise. The process and measurement noise characteristics, respectively, are

$$
\begin{array}{ll}
w \sim N(0, Q) & E\left[(w(k))(w(k))^{T}\right]=Q \\
v \sim N(0, R) & E\left[(v(k))(v(k))^{T}\right]=R
\end{array}
$$

where $R$ is the measurement variance determined by analyzing steady-state data and is

$$
R=\frac{1}{N-1} \sum_{i=1}^{N}\left[\left(y_{i}-\bar{y}\right)^{2}\right]
$$

where $y_{i}$ is the steady-state force measurement at the $i^{\text {th }}$ iteration, $\bar{y}$ is the measurement average, and $N$ is the number of data points. The parameter $Q$ is the model variance and is adjusted with respect to $R$ to tune the filter. Experimentally, a value of $Q=0.05 R$ is selected for this filter. The filter effectively reduces the signal variance by an order of magnitude while preserving the phase and magnitude.

\section{DYNAMIC MODELING}

A series of step tests are conducted to obtain a relationship between axial force and commanded plunge depth for use in designing the axial force controller. The tests are conducted using a 6061-T6 aluminum alloy with material composition: $97.9 \% \mathrm{Al}, 0.60 \% \mathrm{Si}, 0.30 \%$ $\mathrm{Cu}, 1.0 \% \mathrm{Mg}$, and $0.20 \% \mathrm{Cr}$. The tool is tapered, threaded, and contains three flats. The FSW bead-onplate method is conducted during the testing with a single solid $6.35 \mathrm{~mm}$ thick plate. This method does not involve the actual joining of parts; rather, the pin processes solid material. Bead-on-plate welding is commonly used in initial testing to analyze the process without disturbances generated by gaps between the parts. The input process parameters are selected as the plunge depth, traverse rate, and spindle rotation speed. Other factors, such as travel angle and work angle, are held as constant during all runs and, therefore, are not included in the modeling. During each run the transverse rate and spindle speed remain constant, while the plunge depth varies between $4.191-4.716 \mathrm{~mm}$. Note that the shoulder contacts the part at a plunge depth of $4.17 \mathrm{~mm}$. Based on empirical observations, the minimum and maximum traverse rates are 2.0 and $3.2 \mathrm{~mm} / \mathrm{s}$, respectively, and the minimum and maximum spindle rotation speeds are 1300 and $1900 \mathrm{rpm}$, respectively. The selected test conditions are based on a Central Composite Design of Experiments (DOE) and are shown in Table 1.

Measured axial force and plunge depth data, as well as commanded plunge depth data, are obtained for each run at a sample rate of $10 \mathrm{~Hz}$. Results from runs 11 and 12 had poor signal to noise ratios and, thus, were excluded from system modeling. This resulted from the runs being very cold, as shown by their low values of heat index in Table 1. An example of the collected force data is shown in Figure 3. The data indicates a positive correlation between measured axial force and commanded plunge depth. As the commanded plunge depth increases, the axial force increases and eventually reaches a steady value. The initial drop in the measured axial force signal represents an extra transient portion of the process present only in the beginning of the weld. This portion of the data is ignored in modeling to minimize error. Note the first and fifth sements, as well as the second and fourth segments, of measured axial force occur at the same depth but do not have the same average force. This is due to machine geometric errors and stiffness, as well as the amount of flash that is generated. As the amount of flash increases, the depthforce relationship is affected due to less material being present in the weld path to resist the tool.

A static power model that relates the steady-state axial force to the input process parameters is

$$
F_{a}=C v^{\alpha} \omega^{\beta} d^{\gamma}
$$

where $C, \alpha, \beta$, and $\gamma$ are model coefficients. Taking the natural $\log$ of both sides of equation (6)

$$
\ln \left(F_{a}\right)=\ln (C)+\alpha \ln (v)+\beta \ln (\omega)+\gamma \ln (d)
$$

Using the Least Squares method the model parameters are determined to be $C=6.18 \cdot 10^{-2}, \alpha=0.185, \beta=-$ 0.374 , and $\gamma=2.650$.

An empirical second order model of the process and equipment dynamics is

$$
\frac{F_{a}(z)}{U(z)}=v^{\alpha} \omega^{\beta} \frac{b_{1} z+b_{2}}{z^{2}+a_{1} z+a_{2}} z^{-n_{d}}
$$

where $F_{a}(z)$ is the axial force, $U(z)$ is the control signal, $n_{d}$ is the number of delay periods, and $b_{2}, b_{1}, a_{2}$, and $a_{1}$ are model coefficients. The delay is due to an inherent communication delay between the processor implementing the force controller that determines the reference plunge depth and the processor that regulates the plunge position. The control signal and plunge depth are related by

$$
u(z)=d^{\gamma}(z)
$$

Equation (8) is transformed into a difference equation and solved for $F_{a}(k)$

$$
\begin{aligned}
F_{a}(k)= & -a_{1} F_{a}(k-1)-a_{2} F_{a}(k-2) \\
& +v^{\alpha} \omega^{\beta}\left[b_{1} u\left(k-1-n_{d}\right)+b_{2} u\left(k-2-n_{d}\right)\right]
\end{aligned}
$$

A Recursive Least Squares (RLS) algorithm is used to solve for the unknown coefficients of equation (10). The collection of known system inputs and measured system outputs is

$$
\begin{aligned}
& \boldsymbol{\varphi}(k)=\left[\begin{array}{ll}
-f(k-1) & -f(k-2)
\end{array}\right. \\
& \left.u\left(k-1-n_{d}\right) \quad u\left(k-2-n_{d}\right)\right]^{T}
\end{aligned}
$$

where $f(k)$ is the filtered force measurement at iteration $k$. The parameter estimates are

$$
\boldsymbol{\eta}=\left[\begin{array}{llll}
a_{1} & a_{2} & b_{1} & b_{2}
\end{array}\right]^{T}
$$

where the initial values are selected to be unity. The gain matrix is

$$
\mathbf{q}(k)=\mathbf{P}(k-1) \boldsymbol{\varphi}(k)\left[1+\boldsymbol{\varphi}^{T}(k) \mathbf{P}(k-1) \boldsymbol{\varphi}(k)\right]^{-1}
$$


The parameter estimates are

$$
\boldsymbol{\eta}(k)=\boldsymbol{\eta}(k-1)+\mathbf{q}(k)\left[y(k)-\boldsymbol{\varphi}^{T}(k) \boldsymbol{\eta}(k-1)\right]
$$

The matrix covariance is

$$
\mathbf{P}(k)=\left[\mathbf{I}-\mathbf{q}(k) \boldsymbol{\varphi}^{T}(k)\right] \mathbf{P}(k-1)
$$

where the diagonals of the initial covariance matrix are all set to 100. If any of the diagonals of the covariance matrix fall below ten percent of their initial value, a covariance reset is employed to ensure that the covariance matrix does not wind down. The number of delay periods is determined to be $n_{d}=5$. This number is determined based on the average delay observed in the data sets.

The RLS algorithm is applied to runs $1-10$ and the values of the coefficients for each model are determined. These ten sets of coefficients are then averaged to determine the overall system model, as shown in Table 2. The system model is

$$
\frac{F_{a}(z)}{U(z)}=\frac{0.0122 z}{z^{2}-0.8476 z+0.0477} z^{-5}
$$

The open loop transfer function contains two real roots located at 0.787 and $6.06 \cdot 10^{-2}$, corresponding to time constants of 0.418 and $3.57 \cdot 10^{-2} \mathrm{~s}$, respectively. There is a zero at $z=0$. The system is stable and exhibits an overdamped response dominated by the slower time constant. The steady-state gain of equation (16) is $6.10 \cdot 10^{-2}$, which is $1.29 \%$ less than the value of $C$.

Next, the dynamic model is validated through experimental runs using process parameters in the range used to construct the model. Two process parameter sets, $v=2.6 \mathrm{~mm} / \mathrm{s}$ and $\omega=1600 \mathrm{rpm}$, and $v=2.18 \mathrm{~mm} / \mathrm{s}$ and $\omega=1810 \mathrm{rpm}$, are selected for use in validation experiments due to minimal observable flash. Figure 4 shows the axial force measurements taken from varying the plunge depth in a sinusoid manner with a frequency of $0.2 \mathrm{~Hz}$. The maximum error is approximately $6 \%$. Figure 5 shows the axial force measurements taken from varying the plunge depth in a triangular manner at a frequency of $0.1 \mathrm{~Hz}$. The maximum error is approximately $4 \%$. Unlike the previous experiment, the error appears as more evenly distributed about zero. These frequencies of $0.2 \mathrm{~Hz}$ and $0.1 \mathrm{~Hz}$ were chosen based on operator experience due to rate limits imposed on the plunge depth.

\section{CONTROLLER DESIGN}

In this section a controller is designed to regulate the axial force signal. A general tracking controller with constant disturbance rejection is selected and tuned to allow the system to robustly track any desired reference force. General tracking control is a method of combination feed-forward feedback control that theoretically guarantees proper tracking regardless of the reference axial force. The constant disturbance rejection is necessary due to process repeatability issues (i.e., machine stiffness) as a basic general tracking controller does not contain integral action. The controller is implemented in a Smith Predictor-Corrector (SPC) Structure to allow the system to properly account for the pure communication delay. If the inherent communication delay is ignored, the system model becomes

$$
\frac{F_{a}(z)}{U(z)}=\frac{b_{1} z+b_{2}}{z^{2}+a_{1} z+a_{2}}=\frac{b(z)}{a(z)}
$$

The error is

$$
E(z)=F_{R}(z)-F_{a}(z)
$$

where $F_{R}(z)$ is the reference axial force. Equation (18) is rearranged and substituted into equation (17)

$$
v(z) a(z) E(z)=v(z) a(z) R(z)-b(z) U(z)
$$

where $v(z)$ is the disturbance generating polynomial

$$
v(z)=z-1
$$

A dummy control variable, $\mu(z)$, is defined as

$$
\mu(z)=g(z) E(z)=v(z) a(z) R(z)-b(z) U(z)
$$

where $g(z)$ is

$$
g(z)=g_{1} z^{2}+g_{2} z+g_{3}
$$

The coefficients $g_{1}, g_{2}$, and $g_{3}$ are chosen to shape the closed-loop error dynamics. The closed-loop characteristic equation is

$$
z^{3}+\left(a_{1}-1-g_{1}\right) z^{2}+\left(a_{2}-a_{1}-g_{2}\right) z-a_{2}-g_{3}=0
$$

The desired closed-loop system has a pole with a time constant of $\tau=300 \mathrm{~ms}$ and two poles having a natural frequency of $\omega_{n}=30 \mathrm{rad} / \mathrm{s}$ and a damping ratio of $\zeta=$ 0.9 . These closed-loop poles were determined by trial and error and were found to (1) reduce control signal saturation during the transient portion of the response and (2) reduce the natural frequency and increase the damping ratio to decrease the system overshoot, settling time, and oscillations. The desired closed-loop characteristic equation is

$$
z^{3}-1.1076 z^{2}+0.2177 z-0.0245=0
$$

Comparing equations (23) and (24), $g_{1}=-0.74, g_{2}=$ 0.6776 , and $g_{3}=-0.0232$. The controller is implemented in a SPC structure to account for the system communication delay. A block diagram of the system with the general tracking controller implemented in the SPC structure is shown in Figure 6. The signal $E_{1}(z)$ is

$$
E_{1}(z)=\frac{b(z)}{a(z)}\left[1-z^{-5}\right] U(z)
$$

Transforming equation (25) into the difference domain

$$
\begin{aligned}
e_{1}(k)= & -a_{1} e_{1}(k-1)-a_{2} e_{1}(k-2) \\
& +b_{1}[u(k-1)-u(k-1-5)] \\
& +b_{2}[u(k-2)-u(k-2-5)]
\end{aligned}
$$

Combining equations (19), (21), and (25) and transforming into the difference domain, the control signal is 


$$
\begin{aligned}
u(k)= & \frac{b_{2}-b_{1}}{b_{1}} u(k-1)-\frac{b_{2}}{b_{1}} u(k-2)+ \\
& r(k+1)+\frac{a_{1}-1}{b_{1}} r(k)+\frac{a_{2}-a_{1}}{b_{1}} r(k-1)- \\
& \frac{a_{2}}{b_{1}} r(k-2)-\frac{g_{1}}{b_{1}}\left[e(k)-e_{1}(k)\right]- \\
& \frac{g_{2}}{b_{1}}\left[e(k-1)-e_{1}(k-1)\right]- \\
& \frac{g_{3}}{b_{1}}\left[e(k-2)-e_{1}(k-2)\right]
\end{aligned}
$$

\section{EXPERIMENTAL STUDIES}

In this section, a series of bead-on-plate experiments are conducted to validate the general tracking controller. The traverse rate and spindle rotation speed are constant during each experimental run and saturation limits are imposed on the plunge depth between $4.17 \mathrm{~mm}$ and 4.8 $\mathrm{mm}$ to ensure that the tool maintains proper contact with the part. A rate limit on the plunge depth is set at \pm 0.5 $\mathrm{mm} / \mathrm{s}$ to prevent tool breakage. Two runs have reference force signals consisting of a series of step changes, and one run contains a sinusoid reference. The reference force signal magnitudes are selected to utilize a significant portion of the plunge depth range.

The results for an experiment with a constant reference force of $3.7 \mathrm{kN}$ are shown in Figure 7. The standard deviation is $48.1 \mathrm{~N}, 1.23 \%$ of the reference. The second run is conducted by varying the reference axial force in a step-wise manner over a range of different inputs. These results are shown in Figure 8. The average standard deviation over this collection of step inputs is $107.2 \mathrm{~N}$, less than $2.89 \%$ of the reference force. The results of the third run are shown in Figure 9. The reference frequency is $0.2 \mathrm{~Hz}$ and the average absolute value of the errors is $0.1248 \mathrm{kN}$.

The control signal for the first constant input run is shown in Figure 7. Note that significant variations are present in the plunge depth to maintain a constant reference force. These variations are due to machine geometric errors, imperfection of the fixturing method, changes in the thermal boundary conditions as the tool advances along the weld path and a number of other factors. The general tracking controller provides an effective means to compensate for these errors and successfully track a number of desired reference forces.

\section{SUMMARY AND CONCLUSIONS}

Empirical models were constructed from a series of experiments do determine steady-state and dynamic relationships between the input process parameters and the axial force for use in controller design for Friction Stir Welding processes. Following model validation, a general tracking controller with disturbance rejection was designed to robustly track a variety of desired reference axial forces with zero steady-state error. The controller was implemented in a Smith PredictorCorrector structure to account for an inherent communication delay in the FSW system due to the method of controller interrupt.

The controller was validated through a collection of step tests and sinusoid references to demonstrate the ability to track non-constant references. The experimental results demonstrate excellent tracking of all reference signals with minimal error most likely due to the physical limitations of the process (i.e., rate limit due to material stiffness). The standard deviation of the controlled response was found to be approximately one percent of the reference signal magnitude.

\section{REFERENCES}

Cederqvist, L., Bolmsjo, G., and Sorensen, C., 2007, "Adaptive Control of Novel Welding Process to Seal Canisters Containing Sweden's Nuclear Waste Using PID Algorithms," Lunds University.

Kalya, P., 2008, Modeling and Control of Friction Stir Welding, Doctoral Dissertation, Mechanical and Aerospace Engineering Department, Missouri University of Science and Technology.

Zhao, X., Kalya, P., Landers, R.G., and Krishnamurthy, K., 2007, "Design and Implementation of a Nonlinear Axial Force Controller for Friction Stir Welding Processes," American Control Conference, New York, New York, July 11-13.

Table 1: Process Parameters for Step Testing of Plunge Depth.

\begin{tabular}{|c|c|c|c|}
\hline RUN & $\begin{array}{c}\text { Traverse } \\
\text { Rate, } \mathbf{v} \\
(\mathbf{m m} / \mathbf{s})\end{array}$ & $\begin{array}{c}\text { Rotational } \\
\text { Speed, } \boldsymbol{\omega} \\
(\mathbf{r p m})\end{array}$ & $\begin{array}{c}\text { Heat } \\
\text { Index } \\
\left(\mathbf{r p m}^{2} \text { /ipm }\right.\end{array}$ \\
\hline 1 & 2.6 & 1600 & 98.5 \\
\hline 2 & 2.6 & 1900 & 138.8 \\
\hline 3 & 2.18 & 1810 & 150.3 \\
\hline 4 & 3.02 & 1390 & 64.0 \\
\hline 5 & 2.6 & 1600 & 98.5 \\
\hline 6 & 3.02 & 1810 & 108.5 \\
\hline 7 & 2.18 & 1390 & 88.6 \\
\hline 8 & 3.02 & 1390 & 64.0 \\
\hline 9 & 2.18 & 1810 & 150.3 \\
\hline 10 & 2 & 1600 & 128.0 \\
\hline 11 & 3.2 & 1600 & 80.0 \\
\hline 12 & 2.6 & 1300 & 65.0 \\
\hline
\end{tabular}

Table 2: Dynamic Model Coefficients.

\begin{tabular}{|c|c|c|c|c|}
\hline Run & $\mathbf{a}_{\mathbf{1}}$ & $\mathbf{a}_{\mathbf{2}}$ & $\mathbf{b}_{\mathbf{1}}$ & $\mathbf{b}_{\mathbf{2}}$ \\
\hline $\mathbf{1}$ & $-7.42 \mathrm{E}-01$ & $-9.33 \mathrm{E}-02$ & $-1.31 \mathrm{E}-02$ & $2.36 \mathrm{E}-02$ \\
\hline $\mathbf{2}$ & $-1.46 \mathrm{E}+00$ & $5.61 \mathrm{E}-01$ & $-1.99 \mathrm{E}-04$ & $6.63 \mathrm{E}-03$ \\
\hline $\mathbf{3}$ & $-1.05 \mathrm{E}+00$ & $2.37 \mathrm{E}-01$ & $6.73 \mathrm{E}-03$ & $5.24 \mathrm{E}-03$ \\
\hline $\mathbf{4}$ & $-8.70 \mathrm{E}-01$ & $1.15 \mathrm{E}-01$ & $2.56 \mathrm{E}-02$ & $-1.04 \mathrm{E}-02$ \\
\hline $\mathbf{5}$ & $-5.91 \mathrm{E}-01$ & $-2.25 \mathrm{E}-01$ & $2.49 \mathrm{E}-02$ & $-1.43 \mathrm{E}-02$ \\
\hline $\mathbf{6}$ & $-8.32 \mathrm{E}-01$ & $5.23 \mathrm{E}-02$ & $7.66 \mathrm{E}-03$ & $5.59 \mathrm{E}-03$ \\
\hline $\mathbf{7}$ & $-7.20 \mathrm{E}-01$ & $-1.52 \mathrm{E}-03$ & $1.97 \mathrm{E}-02$ & $-2.64 \mathrm{E}-03$ \\
\hline $\mathbf{8}$ & $-7.33 \mathrm{E}-01$ & $-4.27 \mathrm{E}-02$ & $2.19 \mathrm{E}-02$ & $-8.07 \mathrm{E}-03$ \\
\hline $\mathbf{9}$ & $-9.87 \mathrm{E}-01$ & $1.48 \mathrm{E}-01$ & $1.63 \mathrm{E}-02$ & $-7.24 \mathrm{E}-03$ \\
\hline $\mathbf{1 0}$ & $-4.94 \mathrm{E}-01$ & $-2.74 \mathrm{E}-01$ & $1.26 \mathrm{E}-02$ & $1.41 \mathrm{E}-03$ \\
\hline $\mathbf{1 1}$ & n/a & n/a & n/a & n/a \\
\hline $\mathbf{1 2}$ & n/a & n/a & n/a & n/a \\
\hline $\mathbf{a v g}$ & $\mathbf{- 8 . 4 8 E - 0 1}$ & $\mathbf{4 . 7 7 E}-\mathbf{0 2}$ & $\mathbf{1 . 2 2 E}-\mathbf{0 2}$ & $\mathbf{- 2 . 5 7 E - 0 5}$ \\
\hline
\end{tabular}



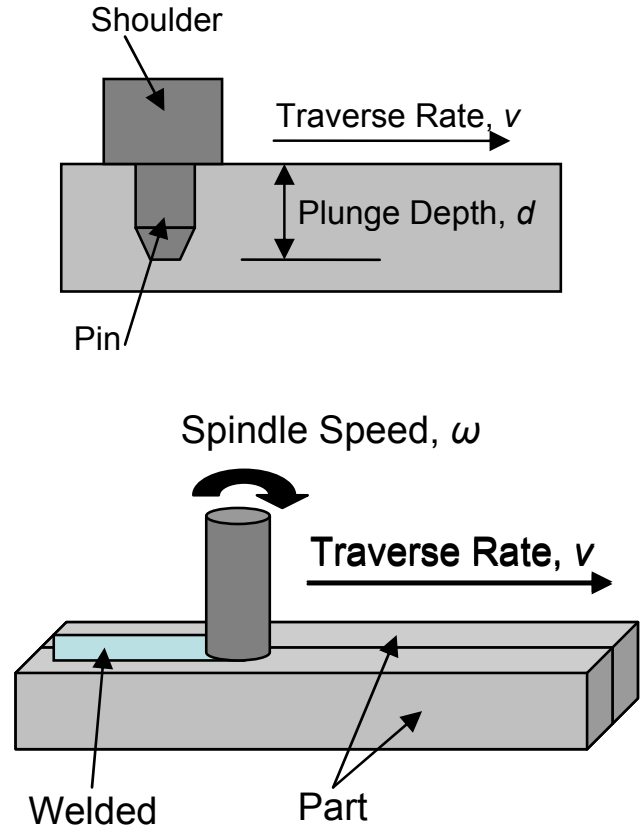

Figure 1: Friction Stir Welding Schematic.

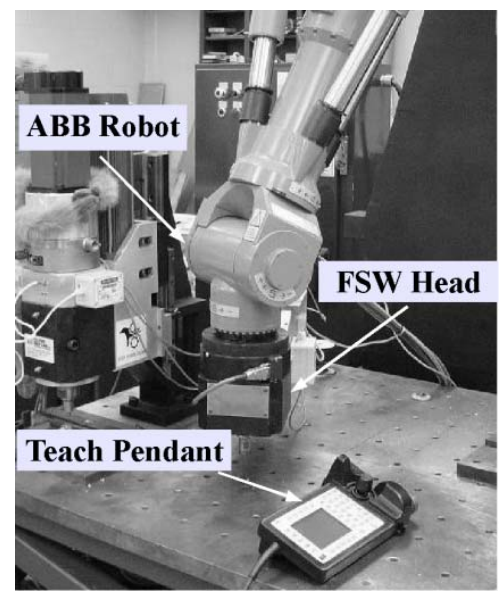

Figure 2: ABB IRB 940 Tricept Robot with FSW Head.
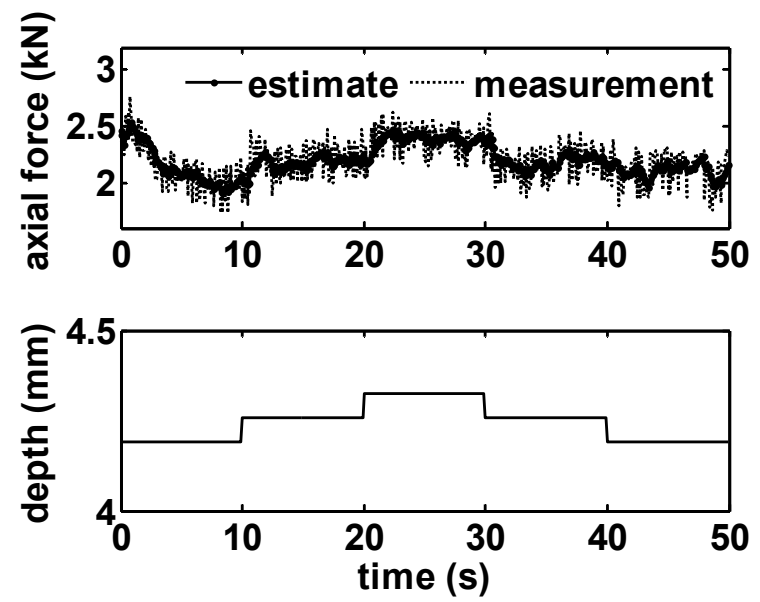

Figure 3: Step Test Force Results and Filter Estimates for Run 1 with $v=2.6 \mathrm{~mm} / \mathrm{s}$ and $\omega=1600$ rpm.

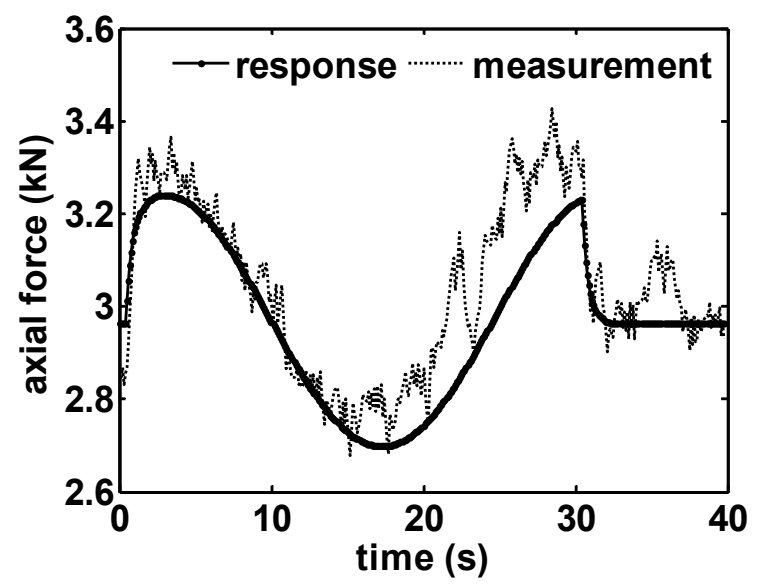

Figure 4: Model Validation Run 1 with $v=2.6 \mathrm{~mm} / \mathrm{s}$ and $\omega=1600 \mathrm{rpm}$.

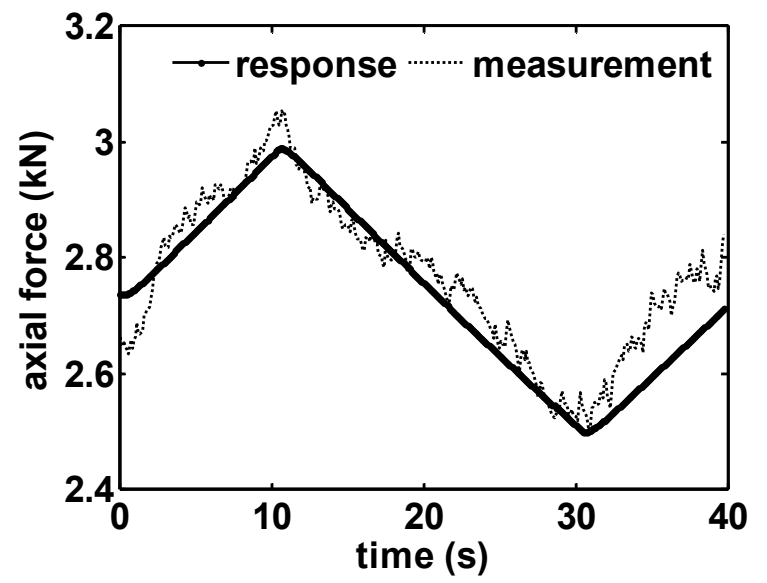

Figure 5: Model Validation Run 2 with $v=2.18 \mathrm{~mm} / \mathrm{s}$ and $\omega=1810 \mathrm{rpm}$.

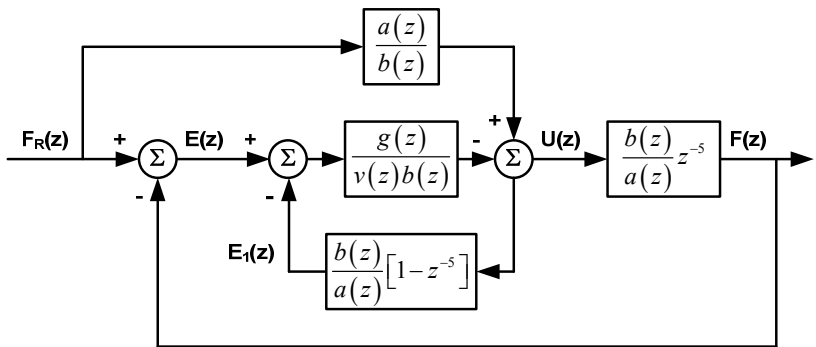

Figure 6: Closed-Loop System Block Diagram with General Tracking Controller in SPC Structure. 

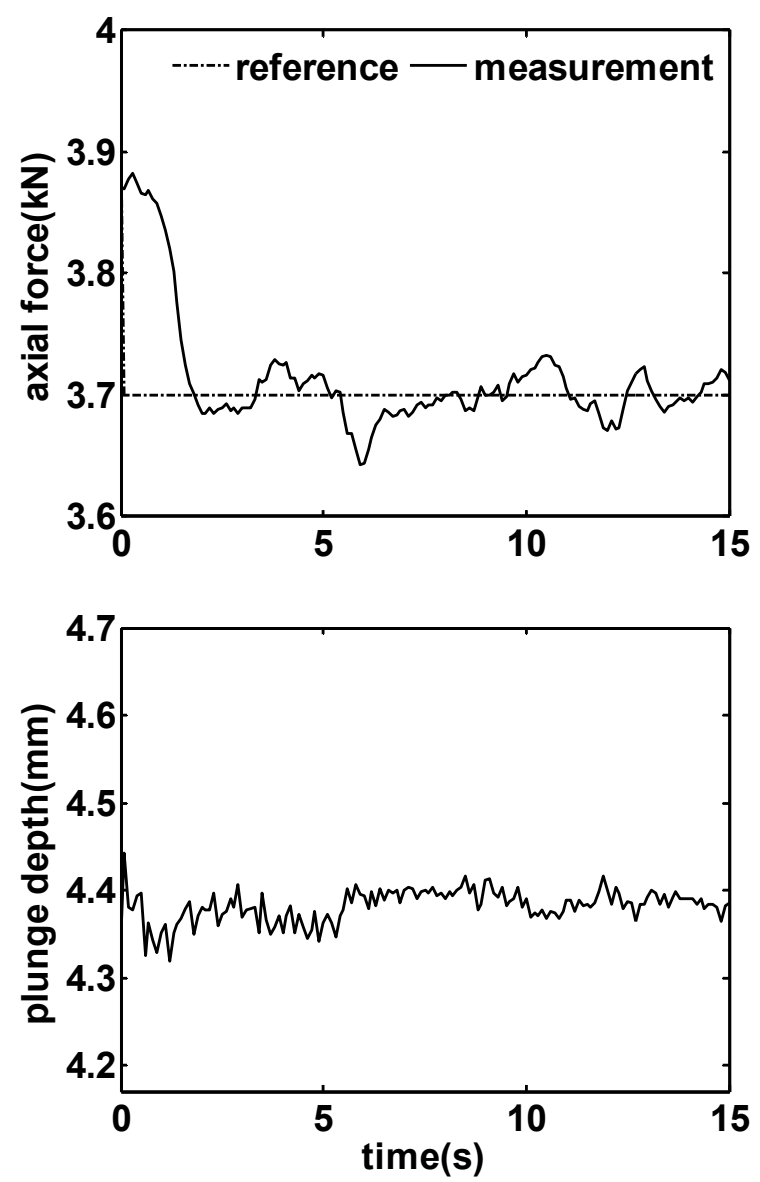

Figure 7: Controller Validation Run 1 with $v=2.18$ $\mathrm{mm} / \mathrm{s}$ and $\omega=1810 \mathrm{rpm}$.

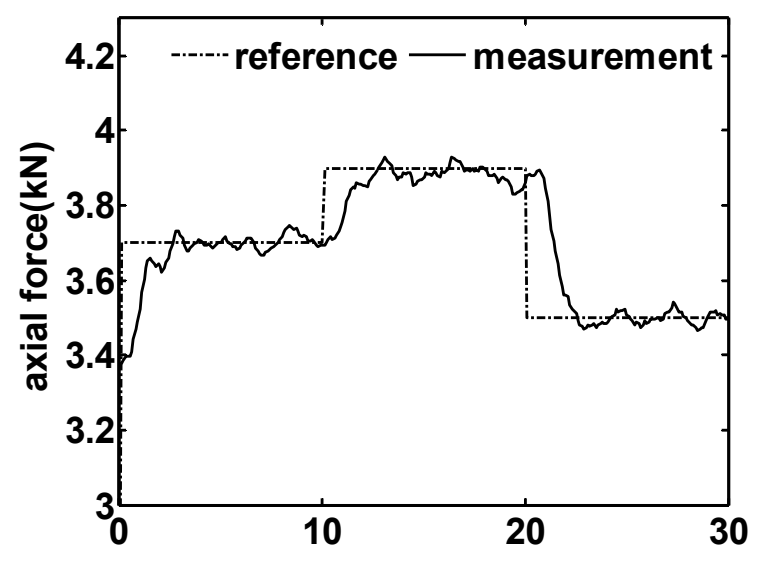

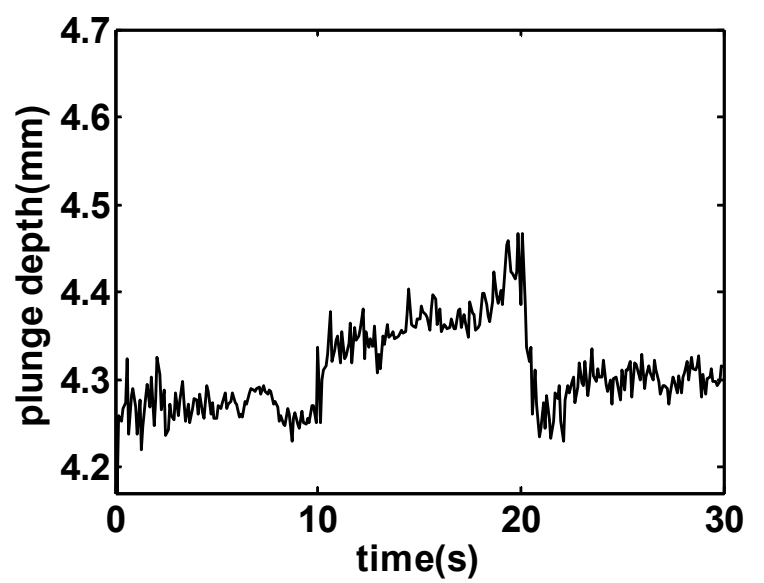

Figure 8: Controller Validation Run 3 with $v=2.6$ $\mathrm{mm} / \mathrm{s}$ and $\omega=1600 \mathrm{rpm}$.
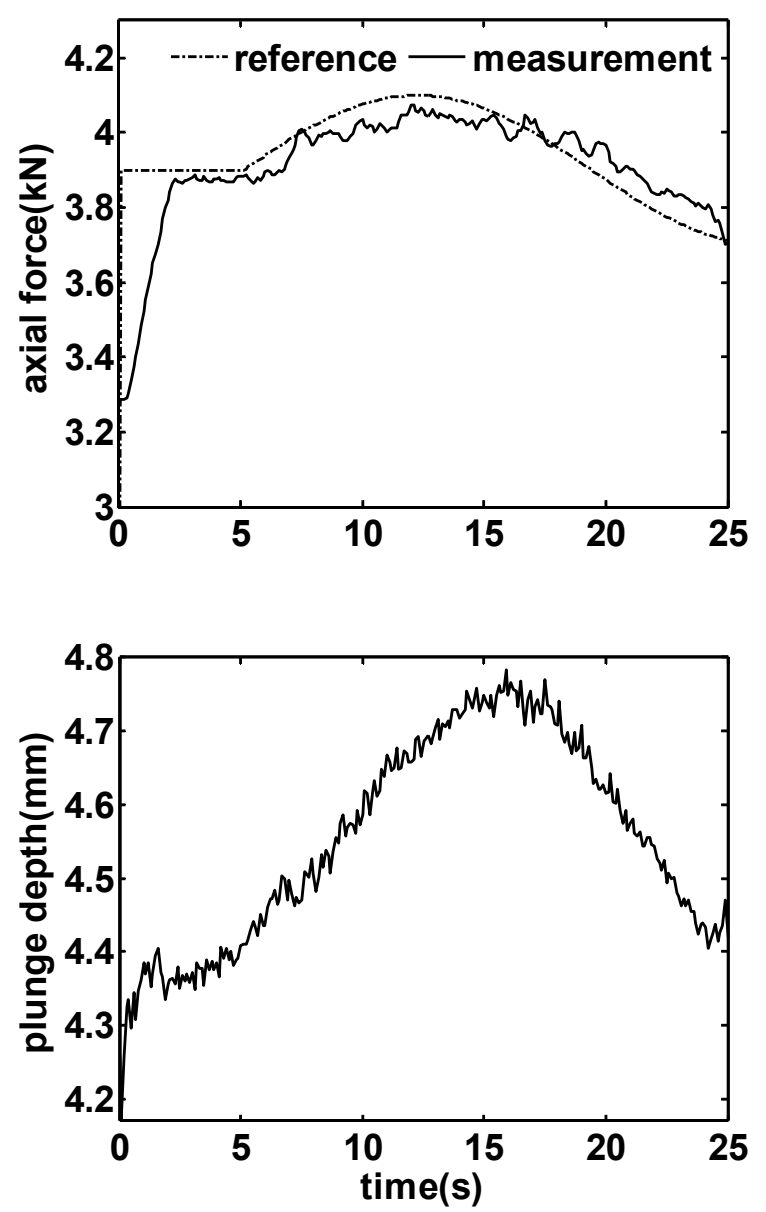

Figure 9: Controller Validation Run 5 with $\mathrm{v}=\mathbf{2 . 1 8}$ $\mathrm{mm} / \mathrm{s}$ and $\omega=1810 \mathrm{rpm}$. 